\title{
Fast and Accurate Pressure-Drop Prediction in Straightened Atherosclerotic Coronary Arteries
}

\author{
Jelle T. C. Schrauwen, ${ }^{1}$ Dion J. Koeze, ${ }^{1}$ Jolanda J. Wentzel, ${ }^{1}$ Frans N. van de Vosse, ${ }^{2}$ \\ Anton F. W. van der Steen, ${ }^{1,3,4}$ and Frank J. H. Gijsen ${ }^{1}$ \\ ${ }^{1}$ Department of Biomedical Engineering, Erasmus Medical Center, 's-Gravendijkwal 230, Faculty Building, Ee 2302, 3000 \\ CA Rotterdam, The Netherlands; ${ }^{2}$ Department of Biomedical Engineering, Eindhoven University of Technology, Eindhoven, \\ The Netherlands; ${ }^{3}$ Department of Imaging Physics, Delft University of Technology, Delft, The Netherlands; and ${ }^{4}$ Shenzhen \\ Institutes of Advanced Technology, Chinese Academy of Sciences, Shenzhen, China
}

(Received 30 April 2014; accepted 5 August 2014; published online 12 August 2014)

Associate Editor Umberto Morbiducci oversaw the review of this article.

\begin{abstract}
Atherosclerotic disease progression in coronary arteries is influenced by wall shear stress. To compute patient-specific wall shear stress, computational fluid dynamics (CFD) is required. In this study we propose a method for computing the pressure-drop in regions proximal and distal to a plaque, which can serve as a boundary condition in CFD. As a first step towards exploring the proposed method we investigated ten straightened coronary arteries. First, the flow fields were calculated with CFD and velocity profiles were fitted on the results. Second, the Navier-Stokes equation was simplified and solved with the found velocity profiles to obtain a pressure-drop estimate $\left(\Delta p_{(1)}\right)$. Next, $\Delta p_{(1)}$ was compared to the pressure-drop from CFD $\left(\Delta p_{\mathrm{CFD}}\right)$ as a validation step. Finally, the velocity profiles, and thus the pressure-drop were predicted based on geometry and flow, resulting in $\Delta p_{\text {geom. }}$. We found that $\Delta p_{(1)}$ adequately estimated $\Delta p_{\mathrm{CFD}}$ with velocity profiles that have one free parameter $\beta$. This $\beta$ was successfully related to geometry and flow, resulting in an excellent agreement between $\Delta p_{\text {CFD }}$ and $\Delta p_{\text {geom }}: 3.9 \pm 4.9 \%$ difference at $R e=150$. We showed that this method can quickly and accurately predict pressure-drop on the basis of geometry and flow in straightened coronary arteries that are mildly diseased.
\end{abstract}

Keywords-Atherosclerosis, Coronary arteries, NavierStokes equation, CFD, Geometry-based, Pressure-drop.

\section{INTRODUCTION}

Ruptures of atherosclerotic plaques in coronary arteries are viewed as the main cause of acute coronary syndrome. ${ }^{2}$ Whether a plaque is rupture-prone is lar-

\footnotetext{
Address correspondence to Jelle T. C. Schrauwen, Department of Biomedical Engineering, Erasmus Medical Center, 's-Gravendijkwal 230, Faculty Building, Ee 2302, 3000 CA Rotterdam, The Netherlands. Electronic mail: j.schrauwen@erasmusmc.nl
}

gely governed by its composition. ${ }^{21,31}$ As increasing evidence shows, plaque composition and rupture location are influenced by wall shear stress (WSS). ${ }^{5,20,23}$ If the WSS over plaques in individual patients could be quantified, it might prove useful in steering treatment, especially in combination with other plaque specific markers for vulnerability, e.g., cap-thickness.

While WSS in coronary arteries cannot directly be measured in vivo, it can be examined with computational fluid dynamics (CFD), which numerically solves the Navier-Stokes (NS) equation. With an accurate reconstruction of the geometry of the coronary artery and appropriate boundary conditions, CFD can compute the entire flow field (e.g. ${ }^{13}$ ). But while software packages can obtain the 3D geometry of the coronary arteries directly, ${ }^{6,26} \mathrm{CFD}$ remains time consuming with conventional technology. To use WSS for decision support during an intervention, CFD must be performed within a limited time frame.

One approach to reducing computational time is to only compute flow dynamics with CFD in the stenosed region, and use reduced-order models for epicardial arteries proximal and distal to the stenosed region. . $^{3,25,27}$ This approach is illustrated in Fig. 1. A 3D reconstruction suited for CFD computations is created based on multi-planar angiography of the stenosed region. The dotted lines represent the epicardial vessels for which reduced-order models based on imaging data could be used (Right panel). At the proximal end of the scheme the proximal pressure ( $p_{\text {prox }}$ ) is imposed that is routinely available during catheterization, and at the distal end a lumped-parameter model is used for the myocardium (Right panel). 


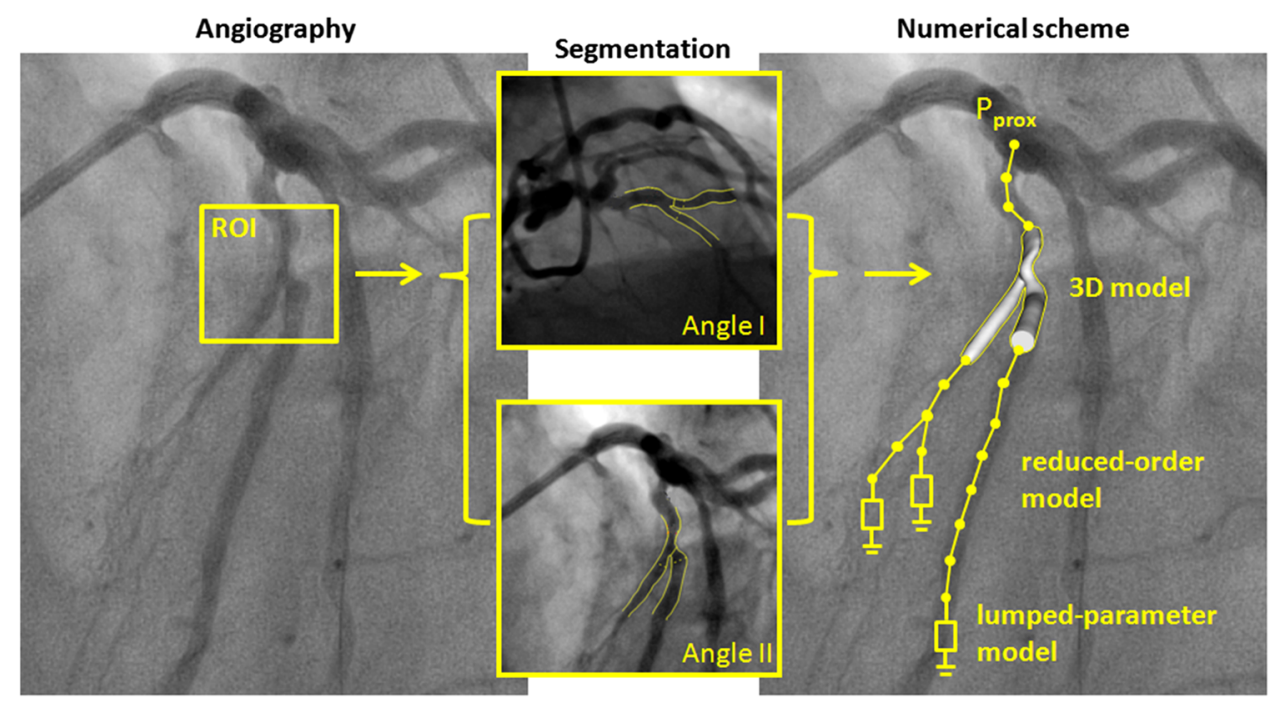

FIGURE 1. Pipeline for online WSS computations. Left panel: Angiography image of coronary artery with stenosed segment, i.e. the region of interest (ROI). Middle panels: Two angiography images of the stenosed region at different angles. Based on these two images a 3D reconstruction suited for CFD computations can be made. Right panel: Illustration of the scheme is superimposed on the angiography image. WSS can be computed with CFD in the 3D model. The dotted lines represent the epicardial vessels for which reduced-order models based on imaging data could be used. The scheme is closed off proximally with pressure $p_{\text {prox}}$, and distally with a lumped-parameter model for the myocardium.

This study focused on finding a reduced-order model based on the geometry of the epicardial arteries for pressure-drop estimation. Past studies presented methods to predict pressure-drop solely on the basis of geometry. In a landmark study, Tsai and Young derived an expression for the pressure-drop over an idealized stenosis as a function of velocity and geometry. ${ }^{34,35}$ This work was later used to estimate the pressure-drop in stenosed human coronary arteries. ${ }^{7}$ Previous work from our group extended those models to incorporate more patient-specific geometrical features. ${ }^{22}$ On average these models performed well, but large deviations can occur per individual vessel.

In this study we propose a method for computing the flow dynamics in regions proximal and distal to a plaque, which can serve as a boundary condition in CFD. The NS equation was simplified and solved with assumed velocity profiles based on the geometry. With this approach we investigated whether the pressuredrop can quickly and accurately be estimated on the basis of geometry and flow.

\section{MATERIALS AND METHODS}

As a first step towards exploring the proposed method we investigated straightened coronary arteries, as straightening reduces the complexity of the flow field. First, the flow fields were computed with CFD and on the results two functions for the velocity pro- files were fitted: $v_{z}^{(1)}$ with one free parameter and $v_{z}^{(3)}$ with three free parameters. Second, the NS equation was simplified and solved with the fitted velocity profiles to obtain the pressure-drop. Third, the resulting pressure-drop estimate was compared with the pressure-drop from CFD as a validation step. Finally, it was found how to predict the velocity profile $v_{z}^{(1)}$, and thus the pressure-drop, on the basis of geometry and flow.

\section{Case Selection}

In the Prospect trial ${ }^{24}$ the three main coronary arteries from patients with acute coronary syndrome were imaged with multi-slice computed tomography and intravascular ultrasound (IVUS). From this study we took the IVUS data of the coronary arteries that did not contain the plaque that had caused the myocardial event. These segments were all affected by atherosclerosis and considered to be mildly diseased. They therefore represent segments proximal and distal to a plaque, since they are often mildly diseased as well. ${ }^{15}$ We used three left anterior descending, five left circumflex and two right coronary arteries with excellent image quality and with the maximal area stenosis within a representative range. The average diameter was $2.8 \pm 0.5 \mathrm{~mm}$ and the average length $44.1 \pm 10.5 \mathrm{~mm}$. The maximal area stenoses in the segments were; $1 \times 20-30 \%, 6 \times 40-50 \%, 1 \times 50$ $60 \%$ and $2 \times 60-70 \%$. All patients gave written informed consent. 


\section{Geometrical Reconstruction and CFD}

In the IVUS images the lumen was segmented. ${ }^{28}$ The centers of the lumen contours were aligned and the distance between the contours was obtained from the IVUS data. From the lumen contours a surface was created (Mevis lab, Bremen, Germany), resulting in the reconstructions of straightened coronary arteries (Fig. 2 top). The side branches were not taken into account in this study. An inlet of five times the inlet radius was added to the reconstruction (VMTK, www.vmtk.org). ${ }^{22}$

The geometries were meshed with GAMBIT (ANSYS, Inc., Canonsburg, PA, USA). Mesh independency studies were conducted, resulting in a surface mesh of triangular elements with an element size of $1.25 \times 10^{-2} \mathrm{~mm}$ and a volume mesh with approximately $10^{6}$ tetrahedral elements. CFD was performed with FIDAP (ANSYS, Inc., Canonsburg, PA, USA) and steady flow was simulated. At the inlet a plug flow was prescribed. The inlet flow was defined on the basis of the Reynolds number ( $R e$ ), which ranged from 25 to 250 with steps of 25 . The outflow conditions were defined as stress free. The blood was modeled as a
Newtonian fluid with a density of $1.0 \times 10^{3} \mathrm{~kg} / \mathrm{m}^{3}$ and a viscosity of $4.0 \times 10^{-3} \mathrm{~Pa}$.

\section{Navier-Stokes Equation}

Flow dynamics is described by the combination of the NS equation and the continuity equation. The NS equation is defined as:

$$
\rho\left(\frac{\partial \vec{v}}{\partial t}+\vec{v} \cdot \nabla \vec{v}\right)=-\nabla p+\mu \nabla^{2} \vec{v} .
$$

In this study we investigated how the NS equation could be simplified to a form in which it can be solved quickly and still provides a good approximation of the pressure-drop. As the pressure-drop was assumed to be predominately in the axial direction, the radial pressure-drop was assumed to be zero and only the axial component of the NS equation had to be solved. Together with assumed axisymmetry and steady flow, the NS equation simplifies to:

$$
\rho\left(v_{\mathrm{r}} \frac{\partial v_{\mathrm{z}}}{\partial \mathrm{r}}+v_{z} \frac{\partial v_{\mathrm{z}}}{\partial \mathrm{z}}\right)=-\frac{\partial p}{\partial \mathrm{z}}+\mu\left(\frac{1}{r} \frac{\partial}{\partial \mathrm{r}}\left(r \frac{\partial v_{\mathrm{z}}}{\partial r}\right)+\frac{\partial^{2} v_{\mathrm{z}}}{\partial z^{2}}\right) .
$$
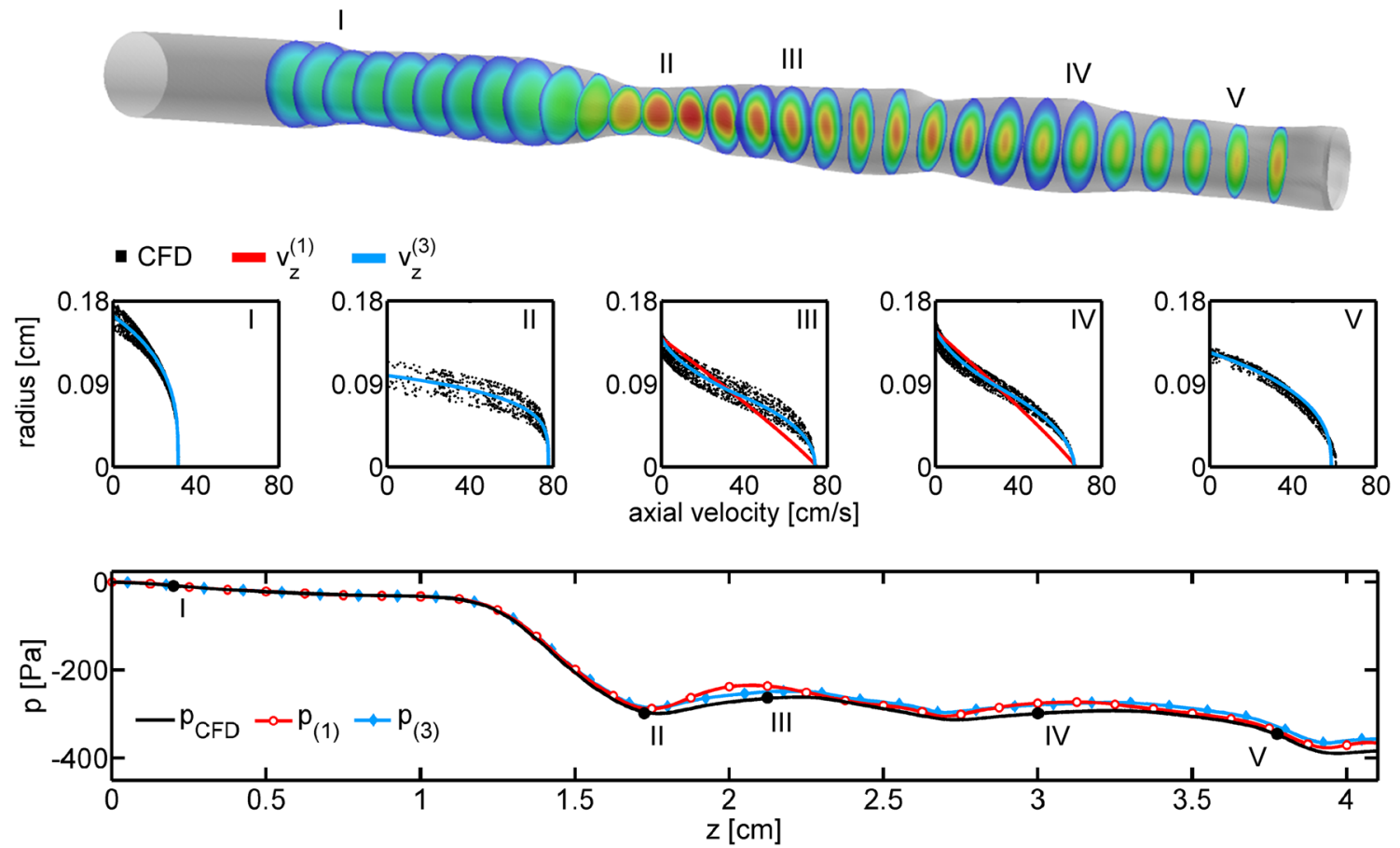

FIGURE 2. Top: A straightened coronary artery based on IVUS data. The iso-planes show the velocity magnitude calculated by CFD. The roman numerals indicate five regions of interest. Middle row: Each panel shows the velocity data from CFD (black dots), with on the horizontal axis the axial velocity and on the vertical axis the distance to center of the lumen. The spread in the velocity data in radial direction is due to the patient-specific lumen which is not circular. In the panels three typical profiles are shown; a parabolic profile in I and V; a plug profile in II; and a sigmoid-shaped profile in II and IV. On the CFD data two functions with different complexity were fitted; $v_{z}^{(1)}$ (blue) with one free parameter and $v_{z}^{(3)}$ (red) with three free parameters. Both $v_{z}^{(1)}$ and $v_{z}^{(3)}$ captured the parabolic and plug profiles. Only $v_{z}^{(3)}$ was able to capture the sigmoid-shaped profiles. Bottom: The pressure-drop as a function axial coordinate. The pressure-drop from CFD (black line) was regarded as the gold standard. The pressure-drop $\Delta p_{(1)}$ was computed with the fits from $v_{z}^{(1)}$, and $\Delta p_{(3)}$ with the fits from $v_{z}^{(3)}$. 
If a form of $v_{z}$ can be assumed that adequately describes the velocity profiles, Eq. (2) can quickly be solved for the axial pressure-drop.

\section{Axial Velocity Profiles}

The velocity profiles that occur in the selected geometries were studied (Fig. 2, middle row) and based on the CFD results, two functions with different complexities were defined to describe the axial velocity profile. First, a simple power-law function was proposed (Fig. 3, Left), previously used by: $:^{12,27,32}$

$$
v_{\mathrm{z}}^{(1)}=v_{\mathrm{z}, \max }\left(1-\left(\frac{r}{a}\right)^{\beta}\right) \text { with } r \in[0, a]
$$

$a=a(z)$ was defined as the radius of the geometry. $a(z)$ was derived as the average radius from the irregular IVUS cross sections. With this $a(z)$ a corresponding $A(z)$ was calculated. The flow $q$ is constant throughout the geometry:

$$
q=\int v_{\mathrm{z}}^{(1)} d A
$$

Equation (3) was rewritten to:

$$
v_{\mathrm{z}}^{(1)}=\frac{q}{\pi a^{2}} \frac{\beta+2}{\beta}\left(1-\left(\frac{r}{a}\right)^{\beta}\right) .
$$

This function has one free parameter $\beta(z)$, which determines the shape of the profile. If $\beta=2$, Eq. (5) results in a Poiseuille profile and a $\beta>2$ results in a more plugged profile. The lower limit of $\beta$ was set to be larger than 1 to ensure a smooth and continuous profile.

As the power-law profile has only one free parameter, it cannot adapt to all the velocity profiles

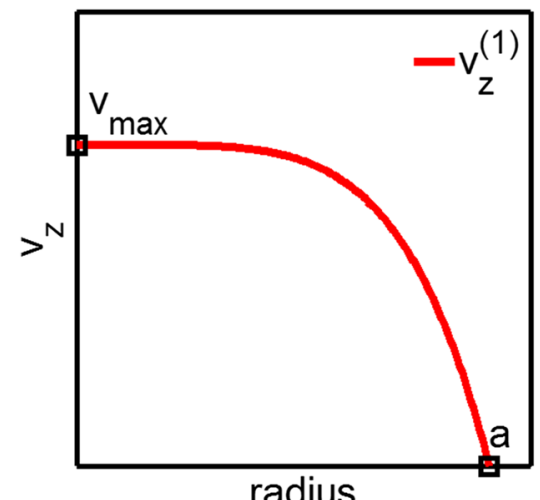

observed in the CFD results, and is especially unable to capture the sigmoid-shaped profiles that can occur in segments with increasing lumen area (Fig. 2, middle row, Panels III and IV). We therefore introduced a second velocity profile $v_{z}^{(3)}$, which has three free parameters $(\beta, c$ and $d)$. The two additional parameters provide more freedom to match the observed profiles. $v_{z}^{(3)}$ is a piecewise function defined by $f_{1}$ and $f_{2}$ (Fig. 3, Right):

$$
v_{z}^{(3)}=\left\{\begin{array}{l}
f_{1} \text { if } 0 \leq r \leq c \\
f_{2} \text { if } c \leq r \leq a
\end{array} .\right.
$$

Point $c(0<c \leq a)$ separates the two functions. The above power-law function described above is retained to describe the profile between the center and point $c$ :

$$
f_{1}=\frac{q}{\pi d^{2}} \frac{\beta+2}{\beta}\left(1-\left(\frac{r}{d}\right)^{\beta}\right) \text { with } r \in[0, c] .
$$

In $f_{1}$, point $d(c<d \leq a)$ determines the transition width in sigmoid-shaped profiles. Function $f_{2}$ describes the profile between point $c$ and the wall:

$$
f_{2}=\gamma_{3} r^{2}+\gamma_{2} r+\gamma_{3} \text { with } r \in[c, a] .
$$

Function $f_{2}$ is able to describe zero flow and reversed flow near the wall. The transition between $f_{1}$ and $f_{2}$ must be continuous and smooth. $f_{2}$ must therefore satisfy:

$$
\begin{aligned}
& f_{2}(a)=0 \\
& f_{2}(c)=f_{1}(c) \\
& \left.\frac{d f_{2}}{d r}\right|_{\mathrm{c}}=\left.\frac{d f_{1}}{d r}\right|_{\mathrm{c}}
\end{aligned}
$$

With these three conditions the values for the three $\gamma$ 's in Eq. (7) were found directly. Functions $f_{1}$ and $f_{2}$ combined lead to $\widehat{v}_{z}^{(3)}$. This profile has to be scaled to the assigned flow $q$. The flow with $\widehat{v}_{z}^{(3)}$ is:

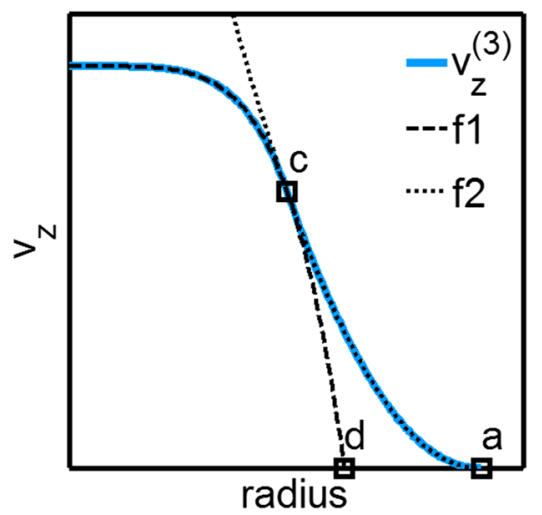

FIGURE 3. The two functions $v_{z}^{(1)}$ and $v_{z}^{(3)}$ were used to describe the axial velocity profiles. Left; the power-law profile $v_{z}^{(1)}$ with one free parameter $\beta$. Right; $v_{z}^{(3)}$ with three free parameters. $v_{z}^{(3)}$ is piece-wise defined by $f_{1}$ and $f_{2}$, that intersect at point c. $f_{1}$ is again a power-law profile that describes the axial velocity in the core. $f_{2}$ is a second-order polynomial that can describe zero flow near the wall. Point d determines the transition width of the sigmoid-shape. 


$$
q_{n}=\int v_{z}^{(3)} d A
$$

With it $\widehat{v}_{z}^{(3)}$ was scaled:

$$
v_{\mathrm{z}}^{(3)}=\widehat{v}_{\mathrm{z}}^{(3)} \frac{q}{q_{\mathrm{n}}} .
$$

\section{Radial Velocity Profiles}

Equation (2) also contains the radial velocity $v_{\mathrm{r}}$. This was derived from the expression for the conservation of mass:

$$
\frac{1}{r} \frac{\partial}{\partial r}\left(r v_{\mathrm{r}}\right)+\frac{1}{r} \frac{\partial v_{\theta}}{\partial \theta}+\frac{\partial v_{z}}{\partial z}=0 .
$$

If $v_{z}$ is known and axisymmetry is assumed, $v_{r}$ becomes the only unknown in Eq. (12). $v_{z}^{(1)}$ enabled us to find an analytical solution for $v_{\mathrm{r}}$ :

$$
\begin{aligned}
v_{\mathrm{r}}= & \frac{q r}{\pi a^{3}} \frac{\beta+2}{\beta}\left(1-\left(\frac{r}{a}\right)^{\beta}\right) \frac{\partial a}{\partial z}+\frac{q r}{\pi a^{2} \beta}\left(\left(\frac{r}{a}\right)^{\beta} \ln \left(\frac{r}{a}\right)\right. \\
& \left.+\frac{1}{\beta}\left(1-\left(\frac{r}{a}\right)^{\beta}\right)\right) \frac{\partial \beta}{\partial z} .
\end{aligned}
$$

In the case of $v_{z}^{(3)}$ the radial velocity was derived numerically.

\section{Numerical Implementation}

With the axial and radial velocities defined, the pressure-drop can be computed directly with Eq. (2). First, Eq. (2) was integrated in the cross sectional direction:

$$
\int \frac{d p}{d z} d A=\int f\left(v_{\mathrm{r}}, v_{\mathrm{z}}\right) d A
$$

$f\left(v_{z}, v_{\mathrm{r}}\right)$ represents the terms in Eq. (2) that contain the velocities. To obtain the pressure-drop, Eq. (14) was subsequently integrated in the axial direction:

$$
\Delta p=\int \frac{d p_{\text {area }}}{d z} d z
$$

where $\frac{d p_{\text {area }}}{d z}$ follows from Eq. (14) after integration. The computations were performed with a Matlab code developed in-house (Matlab 2011b, Mathworks, Natick, U.S.), and generally lasted $10^{-1} \mathrm{~s}$.

\section{Validation}

The pressure-drop from CFD was considered as the gold standard. To validate the method, the estimated pressure-drop was compared to the pressure-drop from
CFD $\left(\Delta p_{\mathrm{CFD}}\right)$. Velocity data from CFD were obtained per cross section with a step size of $\Delta z=0.25 \mathrm{~mm}$. The pressure-drop prediction was independent of the step size at $\Delta z=0.25 \mathrm{~mm} . v_{z}^{(1)}$ and $v_{z}^{(3)}$ were fitted on the CFD data (Fig. 2, middle). The fits of $v_{z}^{(1)}$ gave $\beta_{(1)}$ and those of $v_{z}^{(3)}$ gave $\beta_{(3)}$, c and $\mathrm{d}$. With the fits the pressure-drop was computed with the above described method: $v_{z}^{(1)}$ resulted in $\Delta p_{(1)}$ and $v_{z}^{(3)}$ in $\Delta p_{(3)}$. The comparison between the pressure-drops showed how well the proposed method performs when the velocity profile is optimally captured.

\section{Pressure-Drop Prediction}

The next step was to predict the velocity profiles and thus the parameter $\beta_{(1)}$, to derive the pressure-drop solely on the basis of geometry. Although the addition of the two parameters in $v_{z}^{(3)}$ improved the fits of the velocity profile, it did not improve the pressure-drop prediction (as shown in the result section). Therefore the prediction of the velocity profiles was only performed for $v_{z}^{(1)}$, which has one free parameter $\beta$. At a given location $v_{z}^{(1)}$ depends on the local geometry and on the velocity profile in the preceding cross section. $\beta$ was related to the geometry by the cross sectional area change $\left(\frac{\partial A}{\partial z}\right)$. The $\beta$ in the preceding cross section was noted as $\beta(z-\Delta z)$. With these dependencies, the behavior of $\beta$ was described by a first-order polynomial:

$$
\beta(z)=2+C_{1} \frac{\partial A}{\partial z} \Delta z+C_{2}(\beta(z-\Delta z)-2) \Delta z .
$$

The $\beta$ 's per cross section were obtained from the fits on the CFD results and combined with the corresponding geometrical parameters. Equation (16) was fitted on this data with a least squares method (Matlab 2011b, Mathworks, Natick, U.S.). The average values of $C_{\mathrm{i}}$ were obtained for each $R e$. The relation between $C_{\mathrm{i}}$ and $R e$ was described with a second-order polynomial:

$$
C_{\mathrm{i}}(R e)=D_{1 \mathrm{i}} R e^{2}+D_{2 \mathrm{i}} R e+D_{3 \mathrm{i}} .
$$

By fitting on the $C_{\mathrm{i}}$ 's, the values of the coefficients $D_{\mathrm{ij}}$ in Eq. (17) were obtained. The coefficients $D_{\mathrm{ij}}$ can be used to predict $\beta(\mathrm{z})$ in all geometries.

CFD was performed in ten geometries for ten different $R e$, resulting in 100 cases. To validate the prediction method, leave-one-out cross-validation was used. For this, one case was selected and the coefficients in Eq. (17) were found with the 81 remaining cases consisting of the other nine geometries and nine inlet flows. The coefficients that were found were used to predict $\beta(\mathrm{z})$ for the selected case. $\beta(\mathrm{z})$ was then used 
to directly predict the pressure-drop $\left(\Delta p_{\text {geom }}\right)$. This procedure was repeated for all 100 cases.

Variables were presented as means \pm 1 standard deviation.

\section{RESULTS}

The five panels in the middle row of Fig. 2 illustrate the various profiles that occurred in the straightened coronary arteries. The results of CFD were displayed by plotting the axial velocity components vs. the distance to center of the cross section. The cross-sections are patient-specific and thus not circular, which resulted in the different velocities at a given radius. At the entrance the velocity profile was nearly parabolic (Panel I). Where the lumen narrowed a plug profile developed (Panel II), while a sigmoid-shaped profile appeared in sections where the lumen widened (panel III and IV). $v_{z}^{(1)}$ adequately fitted on the parabolic and plug-shaped profile, but was not able to capture the sigmoid-shaped profile. $v_{z}^{(3)}$ captured all the effects seen in the CFD results.

The pressure-drop in Fig. 2 declined rapidly in the stenosed regions (point II). As the lumen widened again the pressure recuperates somewhat. The behavior of $\Delta p_{\mathrm{CFD}}$ is captured well by both $\Delta p_{(1)}$ and $\Delta p_{(3)}$, except that the pressure recuperation is slightly overestimated. At $R e=150$ (high physiological flow conditions), $\Delta p_{(1)}$ and $\Delta p_{(3)}$ both met the benchmark of $\Delta p_{\mathrm{CFD}}$. They did, however, underestimate $\Delta p_{\mathrm{CFD}}$ increasingly for higher Re (Fig. 4). For the ten geometries, the mean difference between $\Delta p_{(1)}$ and $\Delta p_{(3)}$ never exceeded $3.3 \%$.

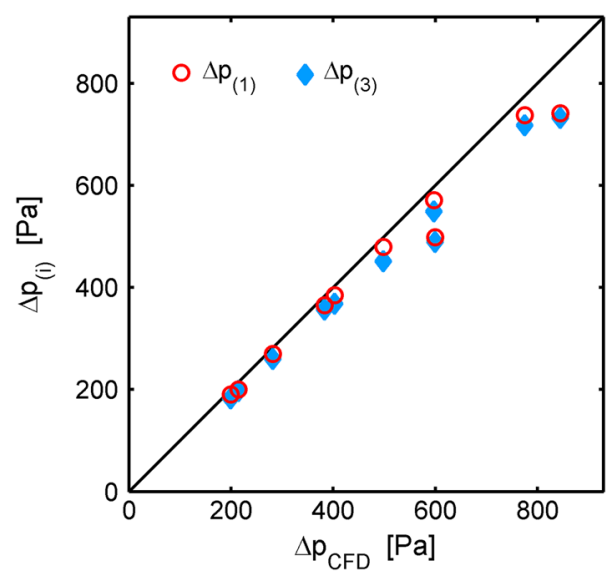

FIGURE 4. Comparison of the found pressure-drops in the ten geometries at $R e=150 . \Delta p_{\mathrm{CFD}}$ is the pressure-drop computed with CFD and $\Delta p_{(i)}$ is the pressure-drop based on fits with either $v_{z}^{(1)}$ or $v_{z}^{(3)}$. A similar pressure-drop was found with $\Delta p_{1}$ (circle) and $\Delta p_{3}$ (diamond), and an good agreement with $\Delta p_{\text {CFD }}$ was found for both.
Since the pressure-drop could accurately be computed with $v_{z}^{(1)}$, only the $\beta$ in $v_{z}^{(1)}$ needed to be predicted and not in $v_{z}^{(3)}$. The behavior of $\beta_{(1)}$ in the geometry from Fig. 2 was first derived from CFD the result and plotted in Fig. 5. The flow was nearly developed at the entrance, resulting in a $\beta_{(1)}$ between 2 and 3 . A plugprofile developed in segments where the lumen narrowed, which caused $\beta_{(1)}$ to increase up to approximately 6 . The sigmoid-shaped profile is not captured well, resulting in a $\beta_{(1)}$ at the limit of 1 . Subsequently, $\beta_{\text {geom }}$ was predicted on the basis of geometry and $R e$, and closely followed $\beta_{(1)}$ (Fig. 5, top). With $\beta_{\text {geom, the }}$ pressure-drop $\Delta p_{\text {geom }}$ was accurately predicted (Fig. 5, bottom). The mean and standard deviation of the coefficients for $\beta_{\text {geom }}$ were found with leave-one-out cross-correlation are presented in Table 1 .

The $\Delta p_{\text {geom }}$ was estimated with high accuracy in all ten geometries. Figure 6 shows that $\Delta p_{\text {geom }}$ nicely meets the benchmark $\Delta p_{\mathrm{CFD}}$ at $R e=100, R e=150$ and $R e=200$. The absolute difference between $\Delta p_{\mathrm{CFD}}$ and $\Delta p_{\text {geom }}$ was small compared to the mean pressuredrop. At $R e=150$, the difference was $35.7 \pm 36.0 \mathrm{~Pa}$ where the mean $\Delta p_{\mathrm{CFD}}$ was $479.7 \mathrm{~Pa}$. At $R e=250$ this increased to $109.9 \pm 124.6 \mathrm{~Pa}$ and a mean $\Delta p_{\mathrm{CFD}}$ of 1025.6 Pa. And thus the relative differences were $3.9 \pm 4.9 \%$ at $R e=150$ and $10.9 \pm 8.2 \%$ at $R e=$ 250. Up to $R e=200$ the relative difference was less than $5 \%$, after $R e=200$ the difference increased exponentially (Fig. 7).

\section{DISCUSSION}

In this study we investigated a method for predicting the pressure-drop in straightened coronary arteries that are mildly diseased. By straightening the coronary arteries the complexity of the flow field was reduced. The NS equation was simplified and assumed velocity profiles were used to compute the pressure-drop. We found that the pressure-drop computed with velocity profiles that have only one free parameter $\beta$, matched the pressure-drop from CFD, which was regarded as the gold standard. This $\beta$ was successfully related to geometry and flow. This approach quickly and accurately predicts the pressure-drop at physiological conditions, and slightly underestimates it at higher $R e$. Combining the model proposed in this study with proximal pressure measurements, a model of the microvascular resistance, and a 3D model for the region of interest, will allow us to compute the shear stress distribution in the 3D segment.

Despite that the momentum balance is not fully taken into account, the pressure-drop was estimated with high accuracy. Only in regions with a relatively large change in lumen area the pressure-drop estimation 

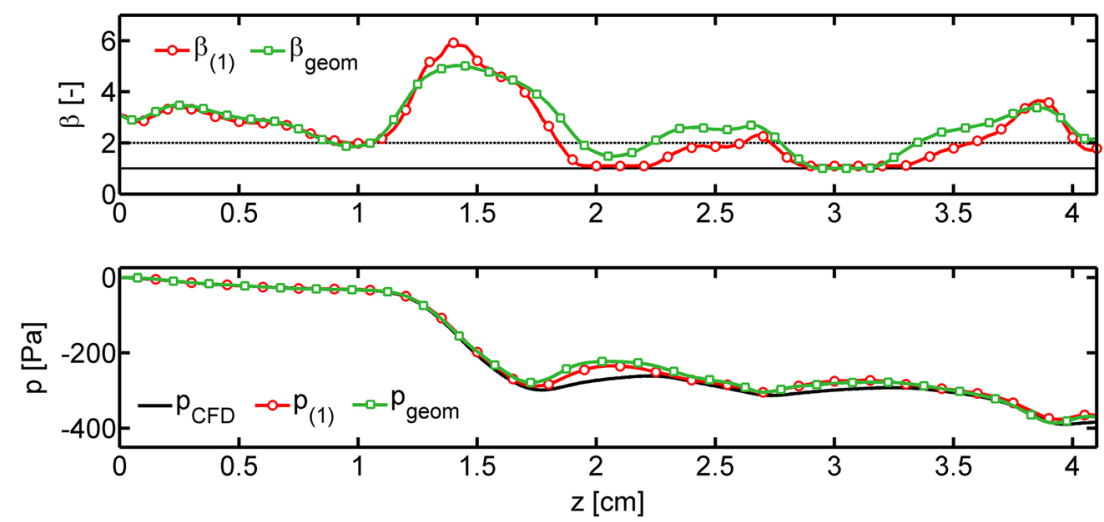

FIGURE 5. Top: The distribution of $\beta$ as a function of the axial coordinate of the geometry shown in Fig. 1. $\beta_{(1)}$ (red) was found by fitting $v_{z}^{(1)}$ on the CFD data. $\beta_{\text {geom }}$ (green) was predicted based on geometry and closely followed $\beta_{(1)}$. Around $z=1.5 \mathrm{~cm}$ the geometry narrows causing a plug profile that resulted in the high values for $\beta$. Around $z=2 \mathrm{~cm}$ and $z=3 \mathrm{~cm}$, the lumen area increases. Here a sigmoid-shaped profile was observed, what results in a $\beta$ close to 1 . Bottom: The pressure-drop as a function of the axial coordinate, where $p_{\text {CFD }}$ (black) is found by CFD, $p_{(1)}\left(\right.$ red) was calculated with the velocity profiles defined by $\beta_{(1)}$, and $p_{\text {geom }}$ (green) by $\beta_{\text {geom. }}$

TABLE 1. Coefficients and the standard deviation of Eq. (17) found with leave-one-out cross-validation.

\begin{tabular}{lrrr}
\hline & \multicolumn{1}{c}{$D_{1 \mathrm{i}}$} & \multicolumn{1}{c}{$D_{2 \mathrm{i}}$} & $D_{3 \mathrm{i}}$ \\
\hline$C_{1}$ & $4.6 \cdot 10^{-4} \pm 9.0 \cdot 10^{-5}$ & $-32.8 \cdot 10^{-2} \pm 2.9 \cdot 10^{-2}$ & $-39.3 \pm 2.3$ \\
$C_{2}$ & $-0.9 \cdot 10^{-4} \pm 1.5 \cdot 10^{-5}$ & $3.2 \cdot 10^{-2} \pm 0.5 \cdot 10^{-2}$ & $35.5 \pm 0.3$ \\
\hline
\end{tabular}

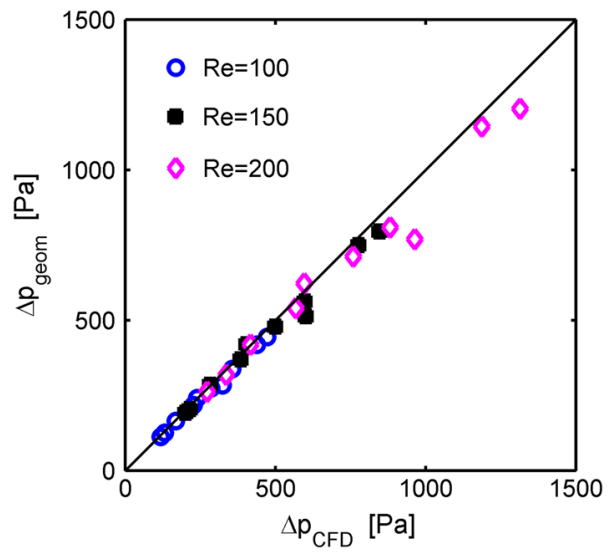

FIGURE 6. The pressure-drop in the ten geometries at $R e=100,150$ and 200 . The $\Delta p_{\mathrm{CFD}}$ was regarded as the gold standard and compared to the pressure-drop predicted based on geometry $\Delta p_{\text {geom }}$. An excellent agreement was found between $\Delta p_{\mathrm{CFD}}$ and $\Delta p_{\text {geom}}$.

deviated from the CFD result. The radial pressuredrop was neglected and it becomes higher in areas with a large change in lumen area, and therefore the estimated total pressure-drops $\Delta p_{(1)}$ and $\Delta p_{(3)}$ deviated more. This explains the deviation seen at higher $R e$ as well. Subsequently we checked if the type of fit on the velocity fields influenced the pressure-drop estimation. Although $v_{z}^{(3)}$ matched the velocity profiles from CFD better than $v_{z}^{(1)}$, it did not improve the pressure-drop estimation much. This means that the deviation of the

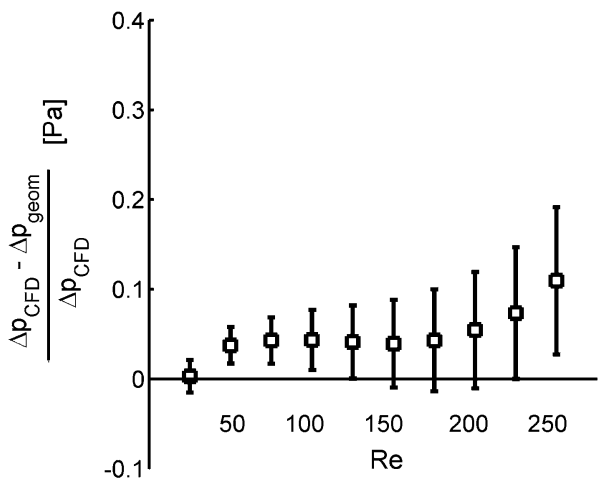

FIGURE 7. The mean relative difference between $\Delta p_{\mathrm{CFD}}$ and the predicted pressure-drop $\Delta p_{\text {geom }}$ in the ten geometries as a function of $R e$. In the physiological range, below $R e=200$, the relative difference is less than $5 \%$. Above $R e=200$, the difference increased exponentially.

pressure-drop estimation can mainly be attributed to neglecting the radial term in the NS equation. Since the axial pressure-drop is much larger than the radial, the pressure-drop is predicted adequately with the simplification of the NS equation for mildly diseased straightened vessels. An estimate for the error associated with neglecting the radial component can be obtained by using the standard deviation of $D_{\mathrm{ij}}$ given in Table 1. A second finding that follows from the accurate pressure-drop estimation with $v_{z}^{(1)}$, is that only one free parameter $\beta$ was needed. Even though $\beta_{(1)}$ sometimes hit the imposed limit of $\beta>1$ the estimation 
remained accurate. Subsequently, $\beta_{\text {geom }}$ was found with a linear combination of a geometrical measure and a preceding $\beta$. In straightened coronary arteries with patient-specific cross sections this gave an excellent prediction of the pressure-drop $\Delta p_{\text {geom }}$.

The proposed method was developed to be used in combination with steady flow simulations. Steady flow is interesting because it is indicative of the WSS that endothelial cells are exposed to over a prolonged period. Clinical studies showed that with this concept relevant information regarding plaque development is obtained. ${ }^{4,24,33}$ Secondly, because parameters used in clinical practice, such as fractional flow reserve, ${ }^{18}$ are based on moving averages. This can be modeled by steady flow simulations as well. Since steady flow simulations only need the geometry at one chosen time point, effects such as cardiac motions or non-rigid walls can be discarded.

This study was designed to help speed up WSS computations in atherosclerotic coronary arteries with CFD. Segments proximal and distal to a plaque can be quantified from imaging data such as angiography or CT. With that imaging data patient-specific boundary condition for CFD can be imposed. For similar purposes, other studies coupled CFD to 1D models. ${ }^{3,12,30}$ These models are based on 1D formulations of the NS equation. ${ }^{1,8,9,11,14,16,19,29}$ These studies were able to obtain valuable time-dependent solutions. However, the models require an estimate for the capacitance of vessel wall, which is difficult to obtain for diseased wall segments. Our model used the $3 \mathrm{D}$ formulation as a starting point and solved it with velocity profiles based on geometrical features. Although the derivation from NS in this study was done differently than the above mentioned models, our model can still be coupled to CFD in a similar fashion. If our method is extended to a formulation that incorporates curvature, it would be interesting to see how well it performs compared to the previously developed 1D-models, since those models cannot distinguish between different degrees of curvature.

A limiting factor for a patient-specific assessment of the WSS is that curvature still has to be implemented. A previous study showed that, for instance, at $R e=175$ this increase was $14.8 \pm 10.0 \% .^{22}$ A first possibility is to choose the same approach as that shown here, but with a curvilinear formulation rather than a cylindrical. While this leads to a mathematically more extensive formulation, it might still be possible to evaluate it. In a curvilinear form an assumed velocity profile can again be defined and subsequently be solved for the pressure-drop. A second, more pragmatic, approach would be to find a geometric measure for the curvature and relate this to the additional pressuredrop the curvature causes. ${ }^{22}$ Apart from including 3D curvature, another limitation for accurate WSS assessment is that the microcirculation has to be taken into account, since this is a key determinant for the flow distribution. However, it cannot be visualized directly with the available imaging techniques. Although several models have already modeled the microcirculation, ${ }^{10,16,17}$ further research is still needed for the resistance distal to diseased coronary arteries. A third requirement is that the lumen segmentations in a clinical setting are based on automatic reconstructions from (bi-plane) angiography, while in this study patient-specific lumen data from IVUS were used which have more detail. The simplification of the lumen might influence the pressure-drop calculations. If so, it is possible to obtain more detail of the lumen with rotational angiography or multiple views. Fourthly, in order for the model to be included in a scheme as presented in Fig. 1, it should be expanded to model the pressure drop in a bifurcation. Next, the spatial derivative of lumen area was taken into account, but the temporal derivative was not. Although we focused on steady flows, it cannot be disregarded that the lumen area changes in time, and that the choice for a fixed lumen area could influence the outcome. Finally, for this study $\beta(0)$ was taken from CFD results. Since this method aims to replace CFD this value will not be available in later applications. We found a linear relation to estimate $\beta(0)$ based on $R e: \beta_{0}(R e)=1.72+$ $R e \times 15 \mathrm{e}-4$. This relation is only based on the ten geometries from this study and might therefore not be generally applicable.

In conclusion, we found that it is possible to quickly and accurately predict pressure-drop on the basis of geometry and flow in straightened coronary arteries that are mildly diseased. Future studies should focus on adding curvature to further advance to patientspecific pressure-drops predictions.

\section{ACKNOWLEDGMENTS}

This research was performed within the framework of the CARISMA-program of STW (Stichting Wetenschap en Techniek), as well as funded by STW.

\section{REFERENCES}

\footnotetext{
${ }^{1}$ Bessems, D., M. Rutten, and F. Van De Vosse. A wave propagation model of blood flow in large vessels using an approximate velocity profile function. J. Fluid Mech. 580:145-168, 2007.

${ }^{2}$ Falk, E., P. K. Shah, and V. Fuster. Coronary Plaque Disruption. Circulation 92(3):657-671, 1995.
} 
${ }^{3}$ Formaggia, L., et al. On the coupling of 3D and 1D NavierStokes equations for flow problems in compliant vessels. Comput. Methods Appl. Mech. Eng. 191:561-582, 2001.

${ }^{4}$ Fukumoto, Y., et al. Localized elevation of shear stress is related to coronary plaque rupture: a 3-dimensional intravascular ultrasound study with in vivo color mapping of shear stress distribution. J. Am. Coll. Cardiol. 51(6):645650, 2008.

${ }^{5}$ Gijsen, F. J. H., et al. Shear stress and advanced atherosclerosis in human coronary arteries. J. Biomech. 46(2):240-247, 2013.

${ }^{6}$ Girasis, C., et al. Novel bifurcation phantoms for validation of quantitative coronary angiography algorithms. Catheter. Cardiovasc. Interv. 77(6):790-797, 2011.

${ }^{7}$ Gould, K. L., K. O. Kelley, and E. L. Bolson. Experimental validation of quantitative coronary arteriography for determining pressure-flow characteristics of coronary stenosis. Circulation 66(5):930-937, 1982.

${ }^{8}$ Huberts, W., et al. A pulse wave propagation model to support decision-making in vascular access planning in the clinic. Med. Eng. Phys. 34(2):233-248, 2012.

${ }^{9}$ Hughes, J. R., and J. Lubliner. On the One-Dimensional Theory of Blood Flow in the Larger Vessels. Math. Biosci. 18:161-170, 1973.

${ }^{10}$ Huo, Y., and G. S. Kassab. Pulsatile blood flow in the entire coronary arterial tree: theory and experiment. Am. J. Phys. Heart Circ. Physiol. 291(3):H1074-H1087, 2006.

${ }^{11}$ Huo, Y., and G. S. Kassab. A hybrid one-dimensional/ Womersley model of pulsatile blood flow in the entire coronary arterial tree. Am. J. Phys. Heart Circ. Phys. 292(6):H2623-H2633, 2007.

${ }^{12} \mathrm{Kim}$, H. J., et al. On coupling a lumped parameter heart model and a three-dimensional finite element aorta model. Ann. Biomed. Eng. 37(11):2153-2169, 2009.

${ }^{13} \mathrm{Kim}$, H. J., K. E. Jansen, and C. A. Taylor. Incorporating autoregulatory mechanisms of the cardiovascular system in three-dimensional finite element models of arterial blood flow. Ann. Biomed. Eng. 38(7):2314-2330, 2010.

${ }^{14}$ Kroon, W., et al. A numerical method of reduced complexity for simulating vascular hemodynamics using coupled 0D lumped and 1D wave propagation models. Comput. Math. Methods Med. 2012:156094, 2012.

${ }^{15}$ Mollet, N. R., et al. Noninvasive assessment of coronary plaque burden using multislice computed tomography. Am. J. Cardiol. 95(10):1165-1169, 2005.

${ }^{16}$ Olufsen, M. S. Structured tree outflow condition for blood flow in larger systemic arteries Structured tree outflow condition for blood flow in larger systemic arteries. Am. J. Physiol. Heart Circ. Physiol. 276:257-268, 1999.

${ }^{17}$ Pahlevan, N. M., et al. A physiologically relevant, simple outflow boundary model for truncated vasculature. Ann. Biomed. Eng. 39(5):1470-1481, 2011.

${ }^{18}$ Pijls, N. H., et al. Measurement of fractional flow reserve to assess the functional severity of coronary-artery stenoses. New Engl. J. Med. 334(26):1703-1708, 1996.

${ }^{19}$ Reymond, P., et al. Validation of a one-dimensional model of the systemic arterial tree. Am. J. Physiol. Heart Circ. Physiol. 297(1):H208-H222, 2009.
${ }^{20}$ Samady, H., et al. Coronary artery wall shear stress is associated with progression and transformation of atherosclerotic plaque and arterial remodeling in patients with coronary artery disease. Circulation 124(7):779-788, 2011.

${ }^{21}$ Schaar, J. A., et al. Terminology for high-risk and vulnerable coronary artery plaques. Report of a meeting on the vulnerable plaque, June 17 and 18, 2003, Santorini, Greece. Eur. Heart J. 25(12):1077-1082, 2004.

${ }^{22}$ Schrauwen, J., et al. Geometry-based pressure drop prediction in mildly diseased human coronary arteries. $J$. Biomech. 47(8):1810-1815, 2014.

${ }^{23}$ Stone, G. W., et al. A prospective natural-history study of coronary atherosclerosis. New Engl. J. Med. 364(3):226235, 2011

${ }^{24}$ Stone, P. H., et al. Prediction of progression of coronary artery disease and clinical outcomes using vascular profiling of endothelial shear stress and arterial plaque characteristics: the PREDICTION Study. Circulation 126(2):172181,2012

${ }^{25}$ Taylor, C. A., and C. A. Figueroa. Patient-specific modeling of cardiovascular mechanics. Ann. Rev. Biomed. Eng. 11:109-134, 2009.

${ }^{26} \mathrm{Tu}, \mathrm{S}$., et al. In vivo assessment of bifurcation optimal viewing angles and bifurcation angles by three-dimensional (3D) quantitative coronary angiography. Int. J. Cardiovasc. Imaging 28(7):1617-1625, 2012.

${ }^{27}$ Van de Vosse, F. N., and N. Stergiopulos. Pulse wave propagation in the arterial tree. Ann. Rev. Fluid Mech. 43(1):467-499, 2011.

${ }^{28}$ Van der Giessen, A. G., et al. The influence of boundary conditions on wall shear stress distribution in patients specific coronary trees. J. Biomech. 44(6):1089-1095, 2011.

${ }^{29}$ Van der Horst, A. et al. Towards patient-specific modeling of coronary hemodynamics in healthy and diseased state. Comput. Math. Methods. Med. 15, 2013.

${ }^{30}$ Vignon-Clementel, I. E., et al. Outflow boundary conditions for three-dimensional finite element modeling of blood flow and pressure in arteries. Comput. Methods Appl. Mech. Eng. 195(29-32):3776-3796, 2006.

${ }^{31}$ Virmani, R., et al. Lessons from sudden coronary death: a comprehensive morphological classification scheme for atherosclerotic lesions. Arterioscler. Thromb. Vasc. Biol. 20:1262-1275, 2000.

${ }^{32}$ Wan, J., et al. A one-dimensional finite element method for simulation-based medical planning for cardiovascular disease. Comput. Methods Biomech. Biomed. Eng. 5(3):195206, 2002.

${ }^{33}$ Wentzel, J. J., et al. Endothelial shear stress in the evolution of coronary atherosclerotic plaque and vascular remodelling: current understanding and remaining questions. Cardiovasc. Res. 96(2):234-243, 2012.

${ }^{34}$ Young, D., and F. Tsai. Flow characteristics in model of aterial stenosis - I steady flow. J. Biomech. 6(4):395-410, 1973.

${ }^{35}$ Young, D., and F. Tsai. Flow characteristics in models of arterial stenoses-II unsteady flow. J. Biomech. 6(1955): 547-559, 1973. 